\title{
New classification criteria developed for research
}

the most
useful
feature of the
classification
system is
its in-built
flexibility

The International Myositis Assessment and Clinical Studies (IMACS) network was established in the late 1990s to develop assessment tools for myositis disease activity. In the course of that work, the need became apparent for reliable classification criteria that were data driven, useful for clinical trial enrolment and research purposes, and were inclusive of adult and paediatric patients. Now, the IMACS-led International Myositis Classification Criteria Project (IMCCP) presents new guidelines that are supported by the ACR and EULAR.

The IMCCP comprised experts from several specialties who treat myositis, including rheumatology, dermatology, neurology and paediatrics. Together, these experts designed the study and validation experiments, revised the list of criteria for assessment and designated the inclusion criteria for cases (a diagnosis of known confidence for at least 6 months before inclusion) and comparators (individuals without myositis). In totaly, 976 patients were enrolled from 47 centres in Europe, the United States and Asia, of which $74 \%$ were adult and $26 \%$ were children; 624 comparators were also included. In looking at different classification techniques, a probabilityscore model was deemed to have superior discriminating performance

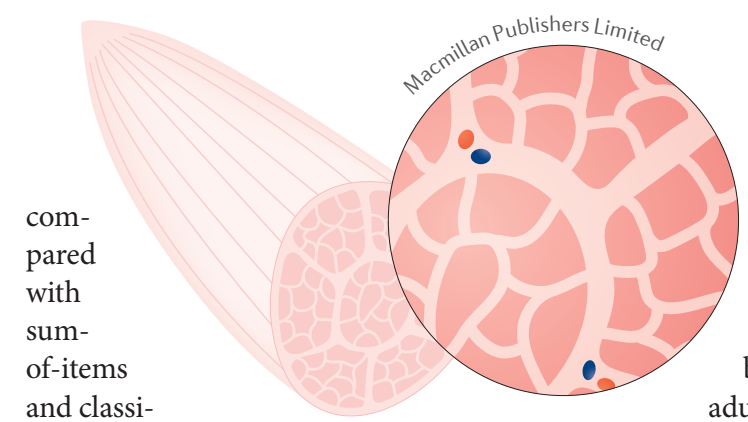

encompass patients with all the major subtypes of myositis dermatomyositis, polymyositis and inclusion body myositis in and classi-

fication-tree approaches and was taken forward and refined.

"The probability-score model gave the best sensitivity and specificity to distinguish myositis based on 16 variables, each given a specific weighted score," explains corresponding author Ingrid Lundberg. The variables include age of onset, type and location of muscle weakness, skin manifestations, laboratory findings and muscle biopsy findings. "A sum of the score gives a probability of having myositis, where the cut-off score of $55 \%$ indicates 'probable' myositis," Lundberg continues. For those individuals who reach a score of $\geq 90 \%$, the classification is deemed 'definite'; patients in the probability score range of $50-55 \%$ are classed as having 'possible' myositis.

Arguably, the most useful feature of the classification system is its in-built flexibility: not all variables need to be assessed to reach a classification of myositis. As the criteria dermatomyositis in children - those with, for example, heliotrope or Gottron rashes can be classified as having dermatomyositis without undergoing muscle biopsy. Additionally, the research network developed a simple classification tree to help researchers determine the subtype of myositis.

Going forward, the classification score will need to be validated in independent cohorts of patients and comparators. "We will also have to update the criteria when we have access to cohorts who have been tested for emerging myositis-specific autoantibodies," explains Lundberg, as the current classification only includes anti-Jo1 autoantibodies.

Mina Razzak, Chief Editor, Nature Reviews Disease Primers

ORIGINAL ARTICLE Lundberg, I. E. et al. 2017 European League Against Rheumatism/American College of Rheumatology classification criteria for adult and juvenile idiopathic inflammatory myopathies and their major subgroups. Arthritis Rheumatol. http://dx.doi.org/10.1002/art.40320 (2017) 